\title{
Incidence and Risk Factors of Hepatic Fibrosis in Psoriatic Patients Receiving Methotrexate with Concomitant Acitretin Therapy and Methotrexate Monotherapy
}

\section{Ploysyne Rattanakaemakorn (iD \\ Prinpat Pinyowiwat $\mathbb{D}$ \\ Wimolsiri lamsumang (D) \\ Kumutnart Chanprapaph (D) \\ Poonkiat Suchonwanit (D)}

Division of Dermatology, Department of Medicine, Faculty of Medicine, Ramathibodi Hospital, Mahidol University, Bangkok, Thailand
Correspondence: Poonkiat Suchonwanit; Kumutnart Chanprapaph

Division of Dermatology, Department of Medicine, Faculty of Medicine,

Ramathibodi Hospital, Mahidol University, 270 Rama VI Road, Ratchathewi, Bangkok,

10400, Thailand

$\mathrm{Tel}+66-2-2011 \mid 141$

Fax +66-2-20I-12II ext 4

Email poonkiat@hotmail.com;

kumutnartp@hotmail.com
Background: The use of methotrexate-acitretin (MTX-ACI) combination therapy in treating psoriasis has been limited due to concerns related to hepatic fibrosis. However, in vitro evidence revealed a protective effect of acitretin in methotrexate (MTX)-induced liver fibrosis. Objective: This study aimed to compare the real-life incidence of hepatic fibrosis in patients with psoriasis receiving MTX-ACI and MTX monotherapy and to investigate factors associated with hepatic fibrosis in MTX-exposed patients.

Methods: A retrospective cohort study was conducted based on a real-life registry containing data on patients with psoriasis who were administered MTX-ACI or MTX between 2008 and 2019 and underwent transient elastography according to cumulative MTX dose of 1.0-1.5 $\mathrm{g}$ and/or 3.5-4.0 g. Time-to-event analysis was performed to determine the cumulative incidence, incidence rate, and factors potentially affecting the occurrence of hepatic fibrosis.

Results: Of the 160 patients, 32 (20\%) were treated with MTX-ACI, and $128(80 \%)$ with MTX alone. Four patients $(12.5 \%)$ in MTX-ACI group and $21(16.4 \%)$ in MTX group developed hepatic fibrosis $(p=0.59)$. There was no statistically significant difference in cumulative incidence $(16 \%$ in MTX-ACI vs $17 \%$ in MTX, $p=0.89)$ and incidence rate $(37$ cases per 1000 person-year in MTX-ACI vs 23 cases per 1000 person-year in MTX; hazard ratio [HR] = $1.07 ; p=0.90$ ) of hepatic fibrosis between the two groups. Diabetes and obesity were identified as significant factors associated with hepatic fibrosis (adjusted HR $=2.40,95 \%$ confidence interval $[\mathrm{CI}]: 1.05-5.51 ; p=0.04$ and adjusted $\mathrm{HR}=3.28,95 \% \mathrm{CI}: 1.18-9.16 ; p=0.02$, respectively) regardless of the cumulative MTX dose.

Conclusion: The incidence of hepatic fibrosis in a real-life clinical situation, determined by transient elastography in patients with psoriasis receiving MTX-ACI, was not increased compared to those receiving MTX monotherapy. Type 2 diabetes mellitus and obesity were identified as risk factors of hepatic fibrosis; hence, patients with these factors receiving longterm MTX therapy should be regularly monitored for this particular event.

Keywords: cirrhosis, combination therapy, drug, liver fibrosis, liver injury, non-alcoholic steatohepatitis

\section{Introduction}

In an era of advancement of biological therapies, methotrexate (MTX) has a central role in the treatment of psoriasis in many countries due to its efficacy and costeffectiveness. $^{1,2}$ Nevertheless, hepatotoxicity is the major side effect occurring in up to $33 \%$ of the patients with long-term MTX therapy and limits its use in psoriatic 
patients with liver impairment. ${ }^{3}$ There is a speculation that MTX may aggravate preexisting non-alcoholic steatohepatitis, which is reported to have a high prevalence in this group of patients. ${ }^{4}$

Acitretin (ACI), a second-generation oral retinoid, is efficacious in the treatment of psoriasis, especially for pustular and erythrodermic subtypes. ${ }^{5}$ It is frequently used in combination with other agents for optimum benefit in treating extensive psoriatic lesions. ${ }^{5,6}$ Furthermore, ACI is accounted as a safe drug for immunocompromised and elderly patients due to lack of immunosuppressive properties. ${ }^{5}$ Although hepatotoxicity can occur in approximately $1.5 \%$ of the patients, it is considered mild, reversible, and often associated with a high-dose regimen. ${ }^{5}$

Combination therapy is a strategy to promote rapid remission of psoriasis by utilizing the synergistic effects of different drugs and reducing potential side effects of high-dose therapy. Although MTX and ACI are efficient in the treatment of psoriasis when used separately, combination of the two may theoretically increase hepatotoxicity. Therefore, the combination of ACI with other hepatotoxic drugs, particularly MTX, is not recommended in consensus guidelines of several countries. ${ }^{7-12}$ Nevertheless, previous studies have revealed the efficacy of the combination therapy in psoriasis with no evidence of increased hepatotoxicity. ${ }^{6,13,14}$ Furthermore, An et al reported that MTX-ACI combination therapy not only enhances treatment effectiveness, but may also reduce MTX-related liver fibrosis by showing less elevation of profibrotic factors in subjects' sera, and lower their expression in hepatic stellate cells compared to MTX monotherapy. ${ }^{15}$ A preliminary study of psoriatic patients treated by MTX-ACI combination therapy also confirmed these findings; however, the lack of a control group limited its conclusion. ${ }^{16}$

Currently, to our knowledge, there is no study comparing the occurrence of hepatic fibrosis in patients with psoriasis treated by MTX-ACI combination therapy and those treated by MTX monotherapy. This study aimed to compare the real-life incidence of hepatic fibrosis in patients with psoriasis receiving MTX-ACI and MTX monotherapy and to investigate factors associated with the development of this side effect in both groups.

\section{Materials and Methods}

This retrospective cohort study was conducted based on a real-life outpatient registry, including patients with psoriasis who attended the dermatology clinic at Ramathibodi Hospital, Bangkok, Thailand. We also included MTX-
ACT-treated subjects from a previous study conducted by some of the authors who matched the inclusion criteria. ${ }^{16}$ The study was approved by the Institutional Review Board for Ethics in Human Research (MURA2019/889) and was performed in accordance with the Helsinki Declaration adopted in 1964. The informed consent to review medical records was exempted by the ethics committee, and data were anonymized prior to analysis.

The medical records of patients with psoriasis who received treatment between January 2008 and December 2019 were retrospectively reviewed. Inclusion criteria included patients with psoriasis aged $\geq 18$ years, receiving systemic treatment with combination therapy of MTX-ACI or MTX alone, and having undergone transient elastography (TE; FibroScan ${ }^{\circledR}$, Echosens ${ }^{\mathrm{TM}}$, Paris, France) following standard recommendation after achieving total accumulative MTX dose of $1.0-1.5 \mathrm{~g}$ and/or 3.5-4.0 g. ${ }^{17}$ TE is a non-invasive method, generating a score in kilopascals $(\mathrm{kPa})$. It has been extensively validated for diagnosing liver fibrosis. ${ }^{18,19}$ MTX in our cohort was prescribed at 5-25 mg weekly, with a minimum cumulative dose of $1.0 \mathrm{~g}$. Therefore, participants included were analyzed based on the administration of a long-term, lowdose MTX regimen. Folic acid was also prescribed concomitantly at $1 \mathrm{mg}$ daily. All patients stopped alcohol consumption during MTX treatment. To estimate the reallife situation, study subjects were included irrespective of their concurrent psoriasis treatments, except for those commencing systemic therapies with hepatotoxicity. Exclusion criteria were patients previously diagnosed and/or possessed conditions associated with hepatic fibrosis, those with concurrent use of hepatotoxic drugs, having aspartate aminotransferase and/or alanine aminotransferase levels greater than twice the upper normal limit, and incomplete medical records.

Demographics, clinical characteristics, disease severity assessments regarding the Psoriasis Area and Severity Index (PASI) score, and treatment details were obtained. PASI score $>10$ was defined as severe psoriasis. Pretreatment alcohol consumption was indicated in patients having average daily alcohol use $\geq$ moderate risk, according to the World Health Organization risk drinking levels. ${ }^{20}$ Obesity was defined as a body mass index of $\geq 30 \mathrm{~kg} / \mathrm{m}^{2}$. Hepatic fibrosis, the outcome of the study, was indicated when the measured value of TE was $>7$ $\mathrm{kPa} .{ }^{18,19}$ Advanced hepatic fibrosis was defined as TE $>$ $8.7 \mathrm{kPa}{ }^{21}$ Based on the analysis, patients were categorized into combined MTX-ACI or MTX groups. Subjects were 
examined at baseline and during subsequent follow-up visits, and followed up until hepatic fibrosis developed or until the end of the study. Collected data were statistically compared between the two groups. Time-to-event analysis was performed to determine the cumulative incidence, incidence rate, and factors potentially affecting the occurrence of hepatic fibrosis in particular patients.

\section{Statistical Analysis}

Statistical analysis was performed using Stata statistical software version 14.2 (Stata Corp, College Station, TX, USA). Categorical variables were reported as proportions and analyzed using chi-square or Fisher's exact test. Continuous variables were expressed as mean \pm standard deviation or median (interquartile range) and analyzed using independent sample $t$-test or Mann-Whitney $U$-test. The person-time analysis was used to determine and compare incidence rates of hepatic fibrosis, which were reported as cases per 1000 person-year. Cox proportional hazards regression was performed to analyze factors associated with the occurrence of hepatic fibrosis. Correlations were reported as hazard ratios (HRs) and $95 \%$ confidence intervals (CIs). Kaplan-Meier method and Log rank test were used to analyze the cumulative incidence of hepatic fibrosis between two treatment groups and between patients with and without risk factors. $P$-value $<0.05$ was considered statistically significant.

\section{Results}

\section{Demographics and Clinical Characteristics}

A total of 242 patients with psoriasis received MTX-ACI therapy or MTX monotherapy. We excluded 58 patients treated with other systemic treatments that may have caused hepatotoxicity and 24 with incomplete medical records. Of the remaining 160 patients, $32(20 \%)$ were treated with MTX-ACI and $128(80 \%)$ with MTX alone. Mean TE values were $5.5 \pm 1.9$ and $5.5 \pm 2.6 \mathrm{kPa}$ in the MTX-ACI and MTX groups, respectively. Demographics and baseline characteristics of participants are demonstrated in Table 1. Four of 32 patients $(12.5 \%)$ in MTX-ACI group (mean TE = $9.2 \pm 2.1 \mathrm{kPa})$ and 21 of 128 patients $(16.4 \%)$ in MTX group (mean TE $=10.1 \pm 2.9 \mathrm{kPa})$ developed hepatic fibrosis. Two $(6.3 \%$, mean $\mathrm{TE}=11.1 \pm 0.1 \mathrm{kPa})$ and $12(9.4 \%$, mean $\mathrm{TE}=$ $11.9 \pm 2.9 \mathrm{kPa})$ in the MTX-ACI and MTX groups showed advanced liver fibrosis, respectively. In comparison with the MTX group, the MTX-ACI group showed significantly lower median age of disease onset (26 [23-78] vs 33
[28-81], $p=0.02)$ and significantly lower percentage of patients with dyslipidemia ( $15.6 \%$ vs $52.3 \%, p<0.001)$, while proportion of patients with pre-treatment alcohol consumption was significantly higher $(15.6 \%$ vs $5.3 \%, p=$ $0.03)$. There was no between-group difference based on gender, type of psoriasis, baseline PASI, percentage of patients with severe psoriasis, the occurrence of hepatic fibrosis and advanced fibrosis, mean TE values, cumulative dose of MTX use, abnormal liver function test at baseline, viral hepatitis (hepatitis B or C), type 2 diabetes mellitus (T2DM), obesity, and history of previous exposure to hepatotoxic agents.

\section{Cumulative Incidence and Incidence Rate of Hepatic Fibrosis in the Study Subjects}

Total follow-up times were 108.65 and 1604.37 personyears in MTX-ACI and MTX groups, respectively. Cumulative incidence of hepatic fibrosis at 5 years was estimated to be $16 \%$ in the MTX-ACI group and $17 \%$ in the MTX group, which revealed no statistically significant difference when determined by Log rank test $(p=0.89$, Figure 1). There was also no statistically significant difference in the incidence rate of hepatic fibrosis between the two groups (37 cases per 1000 person-year in MTX-ACI group vs 23 cases per 1000 person-year in MTX group; $\mathrm{HR}=1.07$, $p=0.90)$.

\section{Factors Associated with Hepatic Fibrosis in the Study Subjects}

Cox proportional hazards method was used to determine factors associated with hepatic fibrosis in the study subjects. Univariate analysis revealed that male gender (HR $=2.70,95 \%$ CI: $1.13-6.47 ; p=0.03$ ), viral hepatitis (HR $=2.88,95 \% \mathrm{CI}: 1.11-7.44 ; p=0.03), \mathrm{T} 2 \mathrm{DM}(\mathrm{HR}=3.65$, 95\% CI: $1.63-8.16 ; p=0.002)$, obesity $(\mathrm{HR}=4.80,95 \%$ CI: $1.79-12.86 ; p=0.002)$, and dyslipidemia $(\mathrm{HR}=3.32$, 95\% CI: $1.35-7.95 ; p=0.007)$ were associated with higher probabilities of hepatic fibrosis. After adjusting for all confounding variables, multivariate regression analysis showed that T2DM (adjusted $\mathrm{HR}=2.40,95 \%$ CI: $1.05-5.51 ; p=0.04$ ) and obesity (adjusted HR $=3.28$, 95\% CI: $1.18-9.16 ; p=0.02)$ increased the probability of developing hepatic fibrosis (Table 2).

Further analysis was performed to test the effect of cumulative MTX dose on each independent risk factor (ie, T2DM and obesity), and results affirmed the association between the two significant factors and hepatic 
Table I Demographics and Clinical Characteristics of Patients at the Time of Methotrexate Initiation

\begin{tabular}{|c|c|c|c|}
\hline \multirow[t]{2}{*}{ Characteristics } & \multicolumn{2}{|c|}{ Treatment Group } & \multirow[t]{2}{*}{$p$-value } \\
\hline & MTX-ATI $(n=32)$ & $\operatorname{MTX}(n=\mid 28)$ & \\
\hline Male, n (\%) & $19(59.4)$ & $63(49.2)$ & 0.30 \\
\hline Age of onset, years, median (IQR) & $26.0(23-78)$ & $33(28-8 I)$ & $0.02 *$ \\
\hline \multicolumn{4}{|l|}{ Type of psoriasis, n (\%) } \\
\hline - Plaque-type psoriasis & $28(87.5)$ & II 4 (89.1) & 0.76 \\
\hline - Pustular psoriasis & $0(0)$ & $2(1.5)$ & 1.00 \\
\hline - Erythrodermic psoriasis & $3(9.4)$ & $5(3.9)$ & 0.19 \\
\hline - Guttate psoriasis & I (3.I) & $7(5.5)$ & 1.00 \\
\hline Baseline PASI, mean (SD) & $8.9(6.1)$ & $8.5(6.3)$ & 0.76 \\
\hline Severe psoriasis, n (\%) & $10(31.2)$ & $36(28.1)$ & 0.82 \\
\hline TE value, $\mathrm{kPa}$, mean (SD) & $5.5 \pm 1.9$ & $5.5 \pm 2.6$ & 0.99 \\
\hline Hepatic fibrosis, n (\%) & $4(12.5)$ & $21(16.4)$ & 0.59 \\
\hline TE value of patients with hepatic fibrosis, $\mathrm{kPa}$, mean (SD) & $9.2 \pm 2.1$ & $10.1 \pm 2.9$ & 0.11 \\
\hline Advanced hepatic fibrosis, $\mathrm{n}(\%)$ & $2(6.3)$ & $12(9.4)$ & 0.57 \\
\hline TE value of patients with advanced hepatic fibrosis, $\mathrm{kPa}$, mean (SD) & $\mathrm{II} . \mathrm{I} \pm 0 . \mathrm{I}$ & $11.9 \pm 2.9$ & 0.12 \\
\hline Cumulative dose of MTX, g, median (IQR) & $2.66(0.65-5.22)$ & $2.08(0-5.64)$ & 0.51 \\
\hline Pre-treatment alcohol consumption, $\mathrm{n}(\%)$ & $5(\mid 5.6)$ & $5(3.9)$ & $0.03^{*}$ \\
\hline Abnormal liver function test, $\mathrm{n}(\%)$ & $0(0)$ & $9(7.0)$ & 0.21 \\
\hline Viral hepatitis B or C, n (\%) & $2(6.2)$ & $17(13.3)$ & 0.37 \\
\hline Diabetes mellitus, n (\%) & $8(25.0)$ & $25(19.5)$ & 0.47 \\
\hline Obesity, n (\%) & $10(31.2)$ & $61(47.7)$ & 0.09 \\
\hline Hyperlipidemia, n (\%) & $5(15.6)$ & $67(52.3)$ & $<0.001 *$ \\
\hline Previous exposure to hepatotoxic agents, $\mathrm{n}(\%)$ & $2(6.2)$ & $18(14.1)$ & 0.37 \\
\hline
\end{tabular}

Notes: *Statistically significant.

Abbreviations: g, gram; IQR, interquartile range; kPa, kilopascals; MTX, methotrexate; MTX-ACl, combination methotrexate and acitretin; PASI, Psoriasis Area and Severity Index; SD, standard deviation; TE, transient elastography.

fibrosis regardless of cumulative MTX dose by showing statistically significant differences in the cumulative incidence of hepatic fibrosis between patients with and without T2DM ( $p=0.025$, Figure 2$)$ and between those with and without obesity ( $p=0.004$, Figure 3$)$.

\section{Discussion}

The present study provided evidence that, based on longterm therapy in a real-life situation, patients with psoriasis regardless of whether they received a combination of MTX and ACI or MTX monotherapy had comparable incidence of hepatic fibrosis determined by TE. We were also able to identify that T2DM and obesity were independent risk factors associated with the occurrence of hepatic fibrosis in particular patients irrespective of cumulative MTX dose.

MTX is considered the conventional systemic therapy in psoriasis, showing its effectiveness in ameliorating extensive skin lesions, but with various adverse effects, especially hepatic fibrosis. ${ }^{22}$ MTX initiates liver fibrosis by prolonging cellular folate depletion and enhancing extracellular adenosine, a profibrotic factor, in hepatic stellate cells, ${ }^{23,24}$ while ACI may accommodate the fibrogenesis process by dysregulating mitochondrial function of hepatic stellate cells, leading to apoptosis and necrosis of these cells. ${ }^{25}$ The occurrence of hepatic fibrosis in MTX therapy is a major concern and variably 


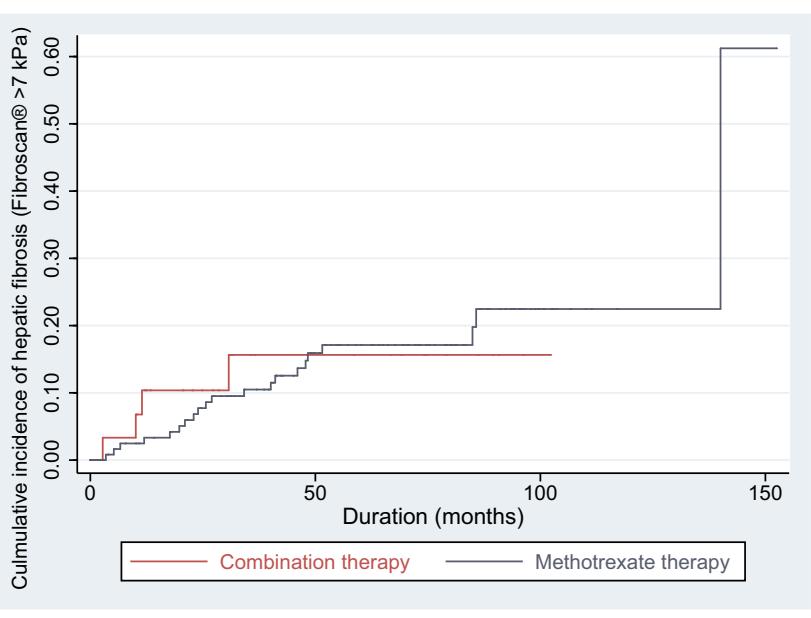

Figure I Cumulative incidence of hepatic fibrosis in patients with psoriasis receiving methotrexate and acitretin combination therapy versus those receiving methotrexate alone.

reported with the overall incidence of $<3 \%$. ${ }^{17,26}$ However, the incidence can be up to approximately $13-34 \%$ in patients with psoriasis receiving long-term MTX therapy with an accumulative dose of at least 1.0 g. ${ }^{27}$ In our cohort, cumulative incidences in both groups, albeit receiving $\mathrm{ACI}$, were consistent with previous reports (16\% in MTX-ACI and $17 \%$ in MTX).

Our study indicated that administration of ACI with MTX is not associated with increased hepatic fibrosis, demonstrating no statistically significant difference in fiveyear cumulative incidence and incidence rate between the treatment groups. Further analysis by Cox regression method supported this finding by showing no association between MTX-ACI therapy and liver fibrosis (Table 2). Our findings support that of a previous study demonstrating the role of retinoic acid in inhibiting liver fibrogenesis. ${ }^{15}$ Retinoic acid signaling may reduce the fibrogenic ability of hepatic stellate cells and synergistically act with peroxisome proliferator-activated receptorgamma in the reversal of hepatic fibrosis. ${ }^{28-30}$

Several risk factors have been reported in association with MTX-induced hepatic fibrosis in patients with psoriasis, including pre-existing liver diseases, alcohol consumption, obesity, dyslipidemia, T2DM, impaired renal function, use of biological agents, and use of other hepatotoxic drugs. $^{31-34}$ Non-alcoholic fatty liver disease (NAFLD) has been also recently identified as a potential

Table 2 Univariate and Multivariate Logistic Regression Analysis of Factors Associated with Hepatic Fibrosis

\begin{tabular}{|c|c|c|c|c|c|c|}
\hline \multirow[t]{2}{*}{ Variables at Baseline } & \multicolumn{3}{|c|}{ Univariate Analysis } & \multicolumn{3}{|c|}{ Multivariate Analysis } \\
\hline & HR & $95 \% \mathrm{Cl}$ & $p$-value & HR & $95 \% \mathrm{Cl}$ & $p$-value \\
\hline MTX-ACI therapy & 1.07 & $0.36-3.13$ & 0.90 & - & - & - \\
\hline Age of onset, years & 1.02 & $0.99-1.05$ & 0.29 & - & - & - \\
\hline Male vs female & 2.70 & $1.13-6.47$ & $0.03 *$ & 1.99 & $0.79-5.01$ & 0.14 \\
\hline Baseline PASI & I.0I & $0.94-1.09$ & 0.78 & - & - & - \\
\hline Severe psoriasis & 1.05 & $0.98-1.13$ & 0.15 & - & - & - \\
\hline Cumulative dose of MTX & 0.99 & $0.99-1.00$ & 0.13 & - & - & - \\
\hline Pre-treatment alcohol consumption & 0.87 & $0.12-6.45$ & 0.89 & - & - & - \\
\hline Abnormal liver function test & 2.62 & $0.49-13.77$ & 0.26 & - & - & - \\
\hline Viral hepatitis B or C & 2.88 & I.II-7.44 & $0.03 *$ & 1.49 & $0.57-3.92$ & 0.42 \\
\hline Diabetes mellitus & 3.65 & $1.63-8.16$ & $0.002 *$ & 2.40 & $1.05-5.51$ & $0.04 *$ \\
\hline Obesity & 4.80 & $1.79-12.86$ & $0.002 *$ & 3.28 & $1.18-9.16$ & $0.02 *$ \\
\hline Hyperlipidemia & 3.32 & I.35-7.95 & $0.007 *$ & 1.73 & $0.67-4.47$ & 0.22 \\
\hline $\begin{array}{l}\text { Previous exposure to hepatotoxic } \\
\text { agents }\end{array}$ & 0.54 & $0.13-2.31$ & 0.41 & - & - & - \\
\hline
\end{tabular}

Notes: *Statistically significant.

Abbreviations: $\mathrm{Cl}$, confidence interval; $\mathrm{HR}$, hazard ratio; $\mathrm{MTX}-\mathrm{ACl}$, combination methotrexate and acitretin; PASI, Psoriasis Area and Severity Index. 


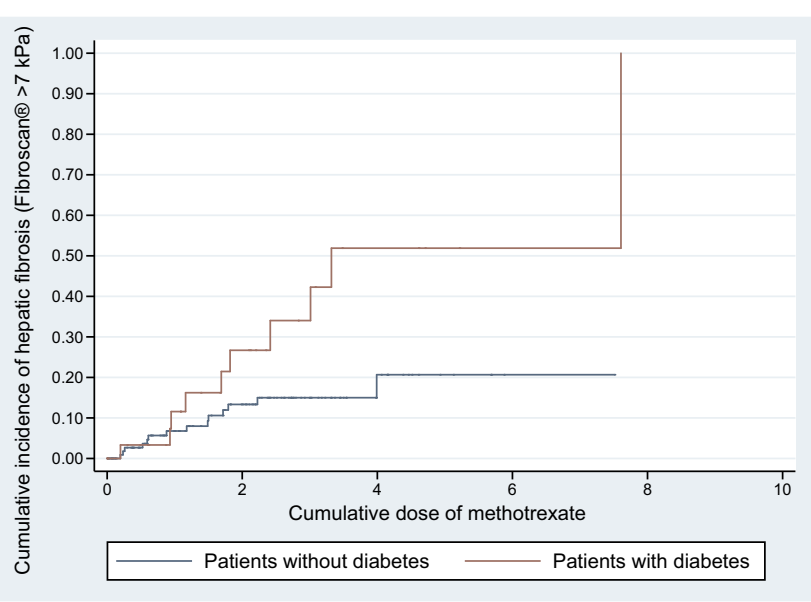

Figure 2 Cumulative incidence of hepatic fibrosis in relation to the cumulative dose of methotrexate in psoriatic patients with and without diabetes.

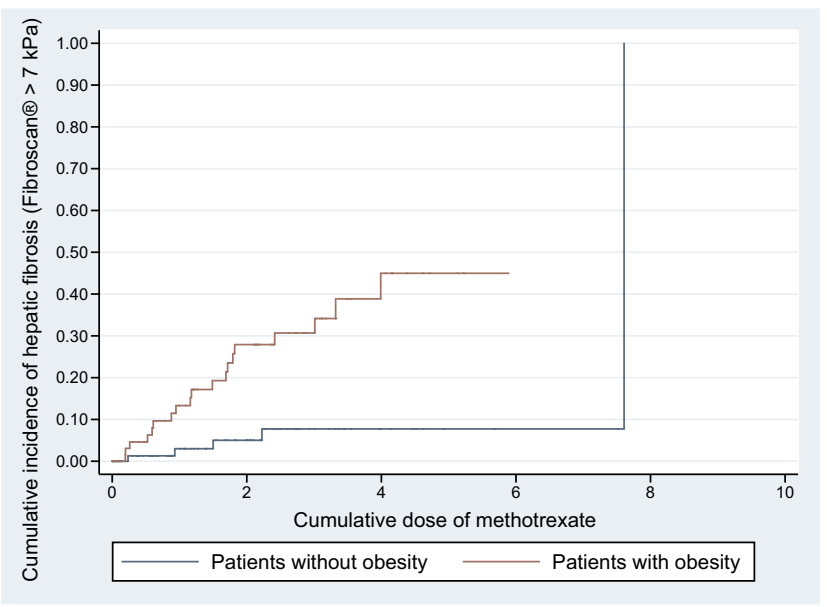

Figure 3 Cumulative incidence of hepatic fibrosis in in relation to the cumulative dose of methotrexate psoriatic patients with and without obesity.

risk factor of drug-induced hepatic fibrosis. ${ }^{35,36}$ On univariate analysis, male gender, viral hepatitis, T2DM, obesity, and dyslipidemia were associated with hepatic fibrosis in our psoriatic patients; however, only T2DM and obesity remained significant on multivariate analysis. Our cohort indicated that patients with $\mathrm{T} 2 \mathrm{DM}$ are at 2.4-fold increased risk of hepatic fibrosis than non-T2DM, and patients with obesity were thrice as likely to develop liver fibrosis than those without. This was in agreement with that of previous studies demonstrating T2DM and obesity as significant predictors, increasing approximately 7 and 2 times the risk of liver fibrosis, respectively, in MTX-treated psoriatic patients. ${ }^{37,38}$

Patients with psoriasis are exposed to multiple liver insults, placing them at high risk of hepatic fibrosis. Several studies reported that T2DM and obesity were prevalent in this group of patients and were factors associated with NAFLD. ${ }^{4,39,40}$ Moreover, patients with psoriasis revealed a higher prevalence of NAFLD compared to general populations. ${ }^{41-43}$ We hypothesized that complex interplay among psoriasis, T2DM, obesity, and NAFLD may play important roles in MTX-induced hepatic fibrosis. Insulin resistance occurring in T2DM initiates hepatic accumulation of triglycerides and subsequent liver steatosis, ${ }^{4}$ whereas obesity demonstrates an increase in non-esterified fatty acids and imbalance between proinflammatory adipocytokines and anti-inflammatory adiponectin, directly promoting liver injury. ${ }^{44}$ Additionally, overproduction of pro-inflammatory cytokines in psoriasis such as tumor necrosis factor-alpha, interleukin (IL)-6, and IL-17 may contribute to the occurrence of NAFLD and progression of liver fibrosis. ${ }^{4-49}$

The association between cumulative dose of MTX and occurrence of liver fibrosis in patients with psoriasis remains inconclusive. Aithal et al reported that the total amount of MTX use increased the frequency of liver fibrosis in MTX-treated psoriasis patients. ${ }^{50}$ Conversely, several studies failed to demonstrate the association between cumulative MTX dose and hepatic fibrosis. $^{37,38,51}$ Rosenberg et al revealed that despite prescribing a lower cumulative MTX dose, patients with psoriasis having risk factors, particularly T2DM or obesity, are at higher risk of developing hepatic fibrosis than those without. ${ }^{52}$ Our results support the latter conclusion, showing no association between cumulative MTX dose and hepatic fibrosis. Furthermore, T2DM and obesity were identified as predictive factors of hepatic fibrosis in our participants regardless of cumulative MTX dose. Whether cumulative MTX dose is associated with hepatic fibrosis in patients with psoriasis or not, those with metabolic syndromes should be routinely screened for NAFLD and liver fibrosis early in their treatment course.

There are several limitations to this study. First, our study was conducted at a tertiary-care single center with a relatively small sample size. Therefore, our results may not be universally applicable. Second, hepatic fibrosis was diagnosed based on the measured value from TE, which may not be as precise as the histopathological diagnosis. However, TE is non-invasive and can accurately diagnose and monitor liver fibrosis. Third, the retrospective design may cause incomplete data collection. Fourth, we did not include MTX-untreated patients as controls; therefore, a causal relationship between MTX administration and worsening of underlying liver diseases could not be 
determined. Finally, participants included in our study were not treatment-naive at enrolment, and we did not completely exclude subjects who may have other riskassociated liver injury, such as pre-treatment alcohol consumption and elevated liver enzymes. Therefore, these undiscovered cofactors may be potential confounding variables; however, the multivariable analysis performed in our study could minimize them. Further prospective studies conducted in a large, multi-center design including biopsy-proven subjects and controls are required to minimize the impact of these limitations and would be useful to affirm our findings.

\section{Conclusion}

Our cohort supports previous in vitro evidence that MTX-ACI combination therapy does not increase the incidence of hepatic fibrosis. We also demonstrated that T2DM and obesity were significant risk factors associated with hepatic fibrosis in long-term MTX-treated psoriatic patients regardless of cumulative MTX dose. Owing to emerging evidence that has suggested the superior efficacy of combination MTX-ACI therapy over MTX alone, this combined regimen could be a promising therapeutic option in patients with psoriasis. However, the choice of combination therapies also depends on safety and tolerability in patients. We recommend that individuals having psoriasis with concomitant metabolic syndrome, especially T2DM and/or obesity, who have been prescribed long-term MTX therapy with high cumulative dosage, should be regularly monitored for potential adverse effects.

\section{Abbreviations}

ACI, acitretin; CI, confidence interval; MTX, methotrexate; MTX-ACI, methotrexate-acitretin; NAFLD, nonalcoholic fatty liver disease; PASI, Psoriasis Area and Severity Index; HR, hazard ratio; TE, transient elastography; T2DM, type 2 diabetes mellitus.

\section{Data Sharing Statement}

All datasets are available from the corresponding author on reasonable request.

\section{Ethics Approval and Consent to Participate}

The study was approved by the Ramathibodi Hospital Review Board for Ethics in Human Research (MURA2019/
889) and was performed in accordance with the Helsinki Declaration adopted in 1964. The informed consent to review medical records was exempted by the ethics committee, and data were anonymized prior to analysis.

\section{Acknowledgments}

We acknowledge the assistance of Dr Kunlawat Thadanipon from the Section of Clinical Epidemiology and Biostatistics, Faculty of Medicine, Ramathibodi Hospital, Mahidol University, in the advisement of statistical analysis.

\section{Funding}

The authors received no financial support for this research.

\section{Disclosure}

The authors report no conflicts of interest in this work.

\section{References}

1. Ng LC, Lee YY, Lee CK, Wong SM. A retrospective review of methotrexate-induced hepatotoxicity among patients with psoriasis in a tertiary dermatology center in Malaysia. Int $J$ Dermatol. 2013;52(1):102-105. doi:10.1111/j.1365-4632.2011.05436.x

2. Miller DW, Feldman SR. Cost-effectiveness of moderate-to-severe psoriasis treatment. Expert Opin Pharmacother. 2006;7(2):157-167. doi:10.1517/14656566.7.2.157

3. Menter A, Korman NJ, Elmets CA, et al. Guidelines of care for the management of psoriasis and psoriatic arthritis: section 4. Guidelines of care for the management and treatment of psoriasis with traditional systemic agents. J Am Acad Dermatol. 2009;61(3):451-485. doi:10.1016/j.jaad.2009.03.027

4. Prussick RB, Miele L. Nonalcoholic fatty liver disease in patients with psoriasis: a consequence of systemic inflammatory burden? $\mathrm{Br}$ J Dermatol. 2018;179(1):16-29. doi:10.1111/bjd.16239

5. Lee CS, Li K. A review of acitretin for the treatment of psoriasis. Expert Opin Drug Saf. 2009;8(6):769-779. doi:10.1517/ 14740330903393732

6. Roenigk HH Jr. Acitretin combination therapy. J Am Acad Dermatol. 1999;41(3 Pt 2):S18-S21. doi:10.1016/S0190-9622(99)70361-0

7. Menter A, Gelfand JM, Connor C, et al. Joint American Academy of Dermatology-National Psoriasis Foundation guidelines of care for the management of psoriasis with systemic nonbiologic therapies. $J A m$ Acad Dermatol. 2020;82(6):1445-1486. doi:10.1016/j. jaad.2020.02.044

8. Ormerod AD, Campalani E, Goodfield MJ. British Association of Dermatologists guidelines on the efficacy and use of acitretin in dermatology. Br J Dermatol. 2010;162(5):952-963. doi:10.1111/ j.1365-2133.2010.09755.x

9. Nast A, Smith C, Spuls PI, et al. EuroGuiDerm guideline on the systemic treatment of Psoriasis vulgaris - Part 1: treatment and monitoring recommendations. J Eur Acad Dermatol Venereol. 2020;34(11):2461-2498. doi:10.1111/jdv.16915

10. Nast A, Amelunxen L, Augustin M, et al. S3 guideline for the treatment of psoriasis vulgaris, update - short version part 1 - systemic treatment. J Dtsch Dermatol Ges. 2018;16(5):645-669.

11. Carretero G, Ribera M, Belinchón I, et al. Guidelines for the use of acitretin in psoriasis. Psoriasis Group of the Spanish Academy of Dermatology and Venereology. Actas Dermosifiliogr. 2013;104 (7):598-616. doi:10.1016/j.ad.2013.01.003 
12. Arnone M, Takahashi MDF, Carvalho AVE, et al. Diagnostic and therapeutic guidelines for plaque psoriasis - Brazilian Society of Dermatology. An Bras Dermatol. 2019;94(2 Suppl 1):76-107. doi:10.1590/abd1806-4841.2019940211

13. Lowenthal KE, Horn PJ, Kalb RE. Concurrent use of methotrexate and acitretin revisited. $J$ Dermatolog Treat. 2008;19(1):22-26. doi:10.1080/09546630701759579

14. Hodulik SG, Zeichner JA. Combination therapy with acitretin for psoriasis. J Dermatolog Treat. 2006;17(2):108-111. doi:10.1080/ 09546630600632984

15. An J, Zhang D, Wu J, et al. The acitretin and methotrexate combination therapy for psoriasis vulgaris achieves higher effectiveness and less liver fibrosis. Pharmacol Res. 2017;121:158-168. doi:10.1016/j. phrs.2017.04.014

16. Pinyowiwat $P$, Rattanakaemakorn $P$, Iamsumang W. 15111 Incidence of hepatic fibrosis in psoriasis patients receiving methotrexate and acitretin combination therapy in a tertiary-care hospital in Thailand. $J$ Am Acad Dermatol. 2020;83(6):AB141. doi:10.1016/j. jaad.2020.06.654

17. Kalb RE, Strober B, Weinstein G, Lebwohl M. Methotrexate and psoriasis: 2009 National Psoriasis Foundation Consensus Conference. $J$ Am Acad Dermatol. 2009;60(5):824-837. doi:10.1016/j. jaad.2008.11.906

18. Park SH, Choe JY, Kim SK. Assessment of liver fibrosis by transient elastography in rheumatoid arthritis patients treated with methotrexate. Joint Bone Spine. 2010;77(6):588-592. doi:10.1016/j. jbspin.2010.02.024

19. Geng XX, Huang RG, Lin JM, Jiang N, Yang XX. Transient elastography in clinical detection of liver cirrhosis: a systematic review and meta-analysis. Saudi J Gastroenterol. 2016;22(4):294-303. doi:10.4103/1319-3767.187603

20. Organization WH. World Health Organization: international guide for monitoring alcohol consumption and related harm; 2000. Available from: https://apps.who.int/iris/bitstream/handle/10665/66529/WHO_ MSD_MSB_00.4.pdf?sequence $=1$ \&isAllowed $=\mathrm{y}$. Accessed January 26, 2021.

21. Wong VW, Vergniol J, Wong GL, et al. Diagnosis of fibrosis and cirrhosis using liver stiffness measurement in nonalcoholic fatty liver disease. Hepatology. 2010;51(2):454-462. doi:10.1002/hep.23312

22. Maybury CM, Jabbar-Lopez ZK, Wong T, Dhillon AP, Barker JN, Smith $\mathrm{CH}$. Methotrexate and liver fibrosis in people with psoriasis: a systematic review of observational studies. $\mathrm{Br} J$ Dermatol. 2014;171(1):17-29. doi:10.1111/bjd.12941

23. Bath RK, Brar NK, Forouhar FA, Wu GY. A review of methotrexate-associated hepatotoxicity. J Dig Dis. 2014;15 (10):517-524. doi:10.1111/1751-2980.12184

24. Cheng HS, Rademaker M. Monitoring methotrexate-induced liver fibrosis in patients with psoriasis: utility of transient elastography. Psoriasis. 2018;8:21-29. doi:10.2147/PTT.S141629

25. Silva FS, Ribeiro MP, Santos MS, Rocha-Pereira P, Santos-Silva A, Custódio JB. Acitretin affects bioenergetics of liver mitochondria and promotes mitochondrial permeability transition: potential mechanisms of hepatotoxicity. Toxicology. 2013;306:93-100. doi:10.1016/j. tox.2013.01.020

26. Belinsky GS, Parke AL, Huang Q, et al. The contribution of methotrexate exposure and host factors on transcriptional variance in human liver. Toxicol Sci. 2007;97(2):582-594. doi:10.1093/toxsci/ kfm067

27. West SG. Methotrexate hepatotoxicity. Rheum Dis Clin North Am. 1997;23(4):883-915. doi:10.1016/S0889-857X(05)70365-3

28. Panebianco C, Oben JA, Vinciguerra M, Pazienza V. Senescence in hepatic stellate cells as a mechanism of liver fibrosis reversal: a putative synergy between retinoic acid and PPAR-gamma signalings. Clin Exp Med. 2017;17(3):269-280. doi:10.1007/ s10238-016-0438-x
29. Suchonwanit P, Hector CE, Bin Saif GA, McMichael AJ. Factors affecting the severity of central centrifugal cicatricial alopecia. Int J Dermatol. 2016;55(6):e338-e343. doi:10.1111/ijd.13061

30. Harnchoowong S, Suchonwanit P. PPAR- $\gamma$ agonists and their role in primary cicatricial alopecia. PPAR Res. 2017;2017:2501248. doi: $10.1155 / 2017 / 2501248$

31. Berends MA, Snoek J, de Jong EM, et al. Liver injury in long-term methotrexate treatment in psoriasis is relatively infrequent. Aliment Pharmacol Ther. 2006;24(5):805-811. doi:10.1111/j.13652036.2006.03047.x

32. Lindsay K, Fraser AD, Layton A, Goodfield M, Gruss H, Gough A. Liver fibrosis in patients with psoriasis and psoriatic arthritis on long-term, high cumulative dose methotrexate therapy. Rheumatology. 2009;48(5):569-572. doi:10.1093/rheumatology/ kep023

33. Rabinowich L, Shibolet O. Drug induced steatohepatitis: an uncommon culprit of a common disease. Biomed Res Int. 2015;2015:168905. doi:10.1155/2015/168905

34. Schmajuk G, Miao Y, Yazdany J, Boscardin WJ, Daikh DI, Steinman MA. Identification of risk factors for elevated transaminases in methotrexate users through an electronic health record. Arthritis Care Res. 2014;66(8):1159-1166. doi:10.1002/ acr. 22294

35. Fromenty B. Drug-induced liver injury in obesity. J Hepatol. 2013;58 (4):824-826. doi:10.1016/j.jhep.2012.12.018

36. Shetty A, Cho W, Alazawi W, Syn WK. Methotrexate hepatotoxicity and the impact of nonalcoholic fatty liver disease. Am J Med Sci. 2017;354(2):172-181. doi:10.1016/j.amjms.2017.03.014

37. Talme T, Nikamo P, Rosenberg P, Stahle M. Transient elastography may improve detection of liver fibrosis in psoriasis patients treated with methotrexate. Acta Derm Venereol. 2017;97(8):952-954. doi:10.2340/00015555-2677

38. Montaudie H, Sbidian E, Paul C, et al. Methotrexate in psoriasis: a systematic review of treatment modalities, incidence, risk factors and monitoring of liver toxicity. J Eur Acad Dermatol Venereol. 2011;25(Suppl 2):12-18. doi:10.1111/j.1468-3083.2011.03991.x

39. Dawwas MF, Aithal GP. End-stage methotrexate-related liver disease is rare and associated with features of the metabolic syndrome. Aliment Pharmacol Ther. 2014;40(8):938-948. doi:10.1111/ apt. 12912

40. Chanprapaph K, Pratumchart N, Limtong P, et al. Dipeptidyl peptidase-4 inhibitor-related bullous pemphigoid: a comparative study of 100 patients with bullous pemphigoid and diabetes mellitus. $J$ Dermatol. 2021;48(4):486-496. doi:10.1111/13468138.15778

41. Ogdie A, Grewal SK, Noe MH, et al. Risk of incident liver disease in patients with psoriasis, psoriatic arthritis, and rheumatoid arthritis: a population-based study. J Invest Dermatol. 2018;138(4):760-767. doi:10.1016/j.jid.2017.10.024

42. Madanagobalane S, Anandan S. The increased prevalence of non-alcoholic fatty liver disease in psoriatic patients: a study from South India. Australas J Dermatol. 2012;53(3):190-197. doi:10.1111/ j.1440-0960.2012.00905.x

43. Candia R, Ruiz A, Torres-Robles R, Chavez-Tapia N, MendezSanchez N, Arrese M. Risk of non-alcoholic fatty liver disease in patients with psoriasis: a systematic review and meta-analysis. $J$ Eur Acad Dermatol Venereol. 2015;29(4):656-662. doi:10.1111/ jdv. 12847

44. Mantovani A, Gisondi P, Lonardo A, Targher G. Relationship between non-alcoholic fatty liver disease and psoriasis: a novel hepato-dermal axis? Int J Mol Sci. 2016;17(2):217. doi:10.3390/ ijms 17020217

45. Gisondi P, Barba E, Girolomoni G. Non-alcoholic fatty liver disease fibrosis score in patients with psoriasis. J Eur Acad Dermatol Venereol. 2016;30(2):282-287. doi:10.1111/jdv.13456 
46. Chanprapaph K, Tubtieng I, Pratumchat N, Thadanipon K, Rattanakaemakorn P, Suchonwanit P. Cutaneous, systemic features and laboratory characteristics of late- versus adult-onset systemic lupus erythematosus in 1006 Thai patients. Lupus. 2021;30 (5):785-794. doi:10.1177/0961203321991920

47. Chanasumon N, Sriphojanart T, Suchonwanit P. Therapeutic potential of bimatoprost for the treatment of eyebrow hypotrichosis. Drug Des Devel Ther. 2018;12:365-372. doi:10.2147/DDDT.S156467

48. Sriphojanart T, Khunkhet S, Suchonwanit P. A retrospective comparative study of the efficacy and safety of two regimens of diphenylcyclopropenone in the treatment of recalcitrant alopecia areata. Dermatol Rep. 2017;9(2):7399. doi:10.4081/dr.2017.7399

49. Rattanakaemakorn P, Phusuphitchayanan P, Pakornphadungsit K, Thadanipon K, Suchonwanit P. Efficacy and safety of 308-nm excimer lamp in the treatment of scalp psoriasis: a retrospective study. Photodermatol Photoimmunol Photomed. 2019;35(3):172-177. doi:10.1111/phpp.12448
50. Aithal GP, Haugk B, Das S, Card T, Burt AD, Record CO Monitoring methotrexate-induced hepatic fibrosis in patients with psoriasis: are serial liver biopsies justified? Aliment Pharmacol Ther. 2004;19(4):391-399. doi:10.1046/j.1365-2036.2004.01819.x

51. Pongpit J, Porntharukchareon S, Kaewduang P, et al. Liver stiffness measurement in psoriasis: do metabolic or disease factors play the important role? Biomed Res Int. 2016;2016:7963972. doi:10.1155/ 2016/7963972

52. Rosenberg P, Urwitz H, Johannesson A, et al. Psoriasis patients with diabetes type 2 are at high risk of developing liver fibrosis during methotrexate treatment. $J$ Hepatol. 2007;46(6):1111-1118. doi:10.1016/j.jhep.2007.01.024

\section{Publish your work in this journal}

Drug Design, Development and Therapy is an international, peerreviewed open-access journal that spans the spectrum of drug design and development through to clinical applications. Clinical outcomes, patient safety, and programs for the development and effective, safe, and sustained use of medicines are a feature of the journal, which has also been accepted for indexing on PubMed Central. The manuscript management system is completely online and includes a very quick and fair peer-review system, which is all easy to use. Visit http://www. dovepress.com/testimonials.php to read real quotes from published authors. 